\title{
Aspectos axiológico-culturales de la ética y el cuidado
}

\author{
María Julia Calvo-Gil ${ }^{1}$, Ricardo Ayala-Valenzuela², Moira Holmqvist-Curimil², Cecilia Molina-Díaz ${ }^{1}$
}

Resumen: El presente trabajo tiene por objetivo descubrir el significado del respeto y el cuidado como dimensiones morales del ejercicio de la enfermería - dentro del sistema de creencias y valores en una cultura específica.

El enfoque teórico de la cuestión está dado por tres elementos que sostienen nuestras concepciones sobre la práctica de enfermería. Con el primero se esboza una discusión acerca de las relaciones entre ética y enfermería; con el segundo se expone el contexto sociohistórico reciente a través del cual el cuidado toma sentido como objeto de estudio de enfermería. El tercero destaca la dimensión de intersubjetividad y co-construcción del cuidado como práctica sociocultural, y subraya la necesidad de sondear y comprender la significación que las dimensiones morales del cuidado adquieren en la condición de sujeto de cuidados.

Fue desarrollada una investigación con enfoque etnográfico, empleando como corpus los textos de entrevistas llevadas a cabo durante el proceso de transculturación del Caring Behaviors Assessment.

Palabras clave: respeto, cuidado, enfermería, conductas de cuidar, cultura

\section{Axiological-cultural aspects of ethics and health care}

\begin{abstract}
The objective of present study is to discover the meaning of respect and care -as moral dimensions of nursing practice- within the system of beliefs and values in a specific culture.

The theoretical focus of the topic is given by three elements which support our conceptions of nursing practice. The first one sketches a discussion about relations between ethics and nursing; the second one shows the recent socio historical context through which care acquires meaning as object of studying nursing. The third one emphasizes the inter subjectivity and co-building dimensions of care as socio cultural practices and highlights the need to seek and understand the meaning that moral dimensions of care acquire in subject condition.

An ethnographic research was developed using as corpus interviews carry out during the process of trans culture on nursing priorities and health care organizations.
\end{abstract}

Key words: respect, care, nursing, care behaviors, culture

\section{Aspectos axiológico-culturais da ética e o cuidado}

Resumo: O presente trabalho tem por objetivo descobrir o significado do respeito e do cuidado como dimensóes morais do exercício da enfermagem - dentro do sistema de crenças e valores numa cultura específica.

O enfoque teórico da questão está dado por três elementos que sustentam nossas concepções sobre a prática de enfermagem. Com o primeiro se esboça uma discussão acerca das relaçóes entre ética e enfermagem; com o segundo se expóe o contexto sócio-histórico recente através do qual o cuidado toma sentido como objeto de estudo da enfermagem. O terceiro destaca a dimensão de intersubjetividade e co-construção do cuidado como prática sócio-cultural, e sublinha a necessidade de sondar e comprender o significado que as dimensôes morais do cuidado adquirem na condição de sujeito de cuidados. Foi desenvolvida uma investigaçáo com enfoque etnográfico empregando como corpus os textos de entrevistas levadas a cabo durante o processo de transculturaçáo do Caring Behaviors Assessment.

Palavras-chave: respeito, cuidado, enfermagem, condutas de cuidar, cultura

\footnotetext{
${ }^{1}$ Enfermera y Matrona. Académica del Instituto de Enfermería, Universidad Austral de Chile, Chile

Correspondencia: mcalvo2@uach.cl

${ }^{2}$ Enfermero (a). Académico de la Escuela de Enfermería, Universidad San Sebastián, Chile
} 


\section{Marco conceptual ${ }^{3}$}

La práctica de la enfermería se caracteriza por una fuerte carga ética, debido a las decisiones envueltas en pro de preservar la dignidad de las personas necesitadas de tratamiento y de cuidado profesional(1). Prácticamente todas las decisiones de enfermería corresponden a una dimensión moral(2), en el entendido de que sus juicios y disposiciones involucran la existencia de otros seres humanos.

Por tanto, el cuidado - en el plano analítico más profundo- emerge de la conciencia acerca del valor que los otros tienen, lo que conduce a desarrollar el respeto, fundamentalmente un respeto referido a la dignidad, no restringido por condiciones específicas de las personas, sino considerando que la necesidad de cuidados es universal y la dignidad y el respeto son rasgos inherentes de la enfermería(3). De acuerdo con estos planteamientos, entonces, desarrollarse como agente de cuidado se relaciona con desarrollar y expandir la propia conciencia o, mejor dicho, la conciencia moral.

Las relaciones entre los conceptos de respeto, cuidado y enfermería se resumen en las proposiciones que nuclean la idea de cuidado, no como continente de una ética específica, sino como una dimensión ética en sí misma(4). Desde esta perspectiva, la práctica de la enfermería es concebida como un ejercicio de naturaleza esencialmente moral, en el que el respeto por las personas y el cuidado son la ética de la profesión. El respeto como una dimensión ética de la enfermería- es evidenciado a través del respeto hacia los sujetos y su familia, hacia sí mismo(a), hacia sus colegas y hacia la profesión, al tiempo que el cuidado como otra dimensión ética- se manifiesta en el cuidado brindado a todos estos actores.

En este mismo sentido, respeto y cuidado son elementos necesarios — aunque no suficientespara la práctica de la enfermería, posiblemente en una correspondencia de uno con otro como antecedente y consecuente, vale decir, en ausencia

3 Esta comunicación corresponde al Proyecto titulado "El valor de cuidar: validación del Caring Behaviors Assessment y lectura del valor social del cuidado". Financiamiento: Dirección de Investigación de la Universidad Austral de Chile (Proyecto DID S-2009-03). de respeto el cuidado no tiene lugar y, por tanto, tampoco la enfermería.

La naturaleza del cuidado ha estado íntimamente vinculada al desarrollo de lo humano como primer acto de vida. De este modo, se entiende como proceso social y como práctica cultural que forma parte de la propia existencia, haciendo posible la conservación de la misma. Por ello, los rasgos esenciales de sobrevivir derivan precisamente del cuidado, que a lo largo de la historia ha recaído mayoritariamente en manos de mujeres, debido a la asociación con su función reproductiva. De esta manera, cuidado y enfermería se combinan en una relación inseparable en que ambos se encuentran contenidos en sí mismos.

Cuidar a alguien es acompañarle(5). No significa imponer un camino ni un itinerario, sino brindar compañía en el marco del respeto a la libertad para escoger. El acompañamiento acompasado y respetuoso posibilita decidir y soportar los sufrimientos.

Cuidar puede ser definido como una ontología(6), una manera de ser, mirar, pensar, compartir y participar con el otro, un fenómeno sociocultural centrado en lo humano y que necesita preparación y conocimiento. Es precisamente la respuesta social al cuidar lo que ha permitido a la disciplina de enfermería construir y reconstruir su historia, en que el cuidado aparece como núcleo central de su ejercicio. Desde una perspectiva epistemológica, el cuidado es producto de la reflexión, de la satisfacción de necesidades del ser humano, y de lo que puede garantizar su bien$\operatorname{estar}(7)$.

Existen distintos elementos que lo caracterizan junto al aspecto concreto de la asistencia y la atención-, referidos a un conjunto de valores, emociones, percepciones, procesos comunicativos, protección y preocupación por el entorno(8). Por contraste, una persona que no cuida es insensible a otra persona como ser único, no es receptiva a los sentimientos de los otros y no necesariamente distingue entre unas personas y otras de un modo significativo(9).

Esto fue planteado ya en las primeras teorías en- 
fermeras. Nightingale, por ejemplo, argumentó la influencia de los aspectos externos en la salud y la enfermedad. Sin embargo, hizo especial énfasis en que las enfermeras debían aplicar la observación y el mayor interés a los pequeños detalles como parte de sus cuidados y aquella que no la practicara simplemente no estaba llamada a serlo. De esta forma, el planteamiento de la fundadora de la enfermería contemporánea sigue vigente para la profesión y la disciplina(10). El ejercicio profesional del cuidado no se confina en los aspectos puramente biologicistas, sino que requiere de un juicio experto, la aplicación del razonamiento en la movilización de recursos, habilidades comunicativas, ética profesional, ejercicio de liderazgo y toma de decisiones fundamentadas en un campo disciplinar.

La práctica de enfermería orientada al cuidado comprende otorgarlo de manera individualizada, como recurso terapéutico que integra habilidades específicas, traduciéndose en un cuidado innovador que une la ciencia y el arte, aunque muchas veces preocuparse de alguien, reforzar sus capacidades, acompañarle en su experiencia de salud-enfermedad, escucharle, educarlo, pasan a ser parte de los llamados cuidados invisibles y/o subvalorados $(11,12)$. Si los aspectos anteriormente señalados no son incorporados se otorgará un cuidado enfermero incompleto, basado en tareas rutinarias y repetitivas.

Leddy y Pepper plantean la incorporación de respeto en la relación enfermero-usuario, para que este último experimente su derecho a existir como persona(13). El enfermero debe proyectar una actitud receptiva que valore los sentimientos, opiniones, individualidad y carácter único del usuario. El cuidado se construye entonces sobre la base del respeto, la igualdad y reciprocidad.

La perspectiva de los sujetos receptores de cuidados cobra tal importancia que hoy es un factor crucial en la concepción que ellos tienen sobre los aspectos cualitativos de la asistencia(14-16). Si bien los enfermeros valoran habitualmente las actividades técnicas y procedimientos como su primera obligación(17), parece claro que los individuos necesitan énfasis en el involucramiento con ellos, la cercanía y dar tiempo y atención para manifestar ideas y sentimientos, especialmente en situaciones en que experimentan vulnerabili$\operatorname{dad}(18-22)$.

El arte de las enfermeras está en cómo usar y adaptar creativamente el conocimiento experto que se ha construido científicamente(23), lo que demanda ser receptivo a las necesidades que otros viven desde su mundo subjetivo. Así, la interacción y la conexión relacional dan significado a una realidad co-construida intersubjetivamente en el discurrir de las experiencias de cuidar y ser cuidado(24).

\section{Objetivo}

El objetivo de este trabajo es descubrir el significado que personas hospitalizadas asignan al respeto y al cuidado - como dimensiones morales del ejercicio de la enfermería- dentro del sistema específico de creencias y valores que se manifiestan mediante la práctica discursiva de una cultura específica. El planteamiento de este objetivo se sustenta en el supuesto de que la producción de discursos abre camino al mundo de significados compartidos de una colectividad, por ende, al entramado del espacio simbólico que los individuos construyen como actores de realidades intersubjetivas y múltiples, y que mediante su análisis es posible acceder a este espacio.

\section{Método}

En atención a este objetivo, desarrollamos una investigación cualitativa de enfoque etnográfico, llevada a cabo en un hospital público chileno. Como principal registro etnográfico empleamos discursos obtenidos de entrevistas llevadas a cabo durante el proceso de transculturación del Caring Behaviors Assessment (CBA) (25) en Chile, con la participación de 35 adultos seleccionados mediante muestreo de conveniencia, previo proceso de información y firma de formulario de consentimiento. El proyecto fue llevado a cabo a principios de 2010 con el objeto de identificar conductas que los enfermeros pueden mostrar para hacer notar que cuidan de otros.

Para contextualizar al lector, es preciso señalar que 
esta escala contiene indicadores empíricos de la Filosofía del Cuidado Humano(26-28), corriente influida por el existencialismo, la fenomenología, el interaccionismo simbólico y la psicología transpersonal. El constructo "cuidado" es entendido a partir de siete dimensiones: humanismo, fe, esperanza y sensibilidad; ayuda y confianza; expresión de sentimientos positivos y negativos; enseñanzaaprendizaje; soporte y ambiente de protección; asistencia en las necesidades humanas; fuerza existencial, fenomenológica y espiritual.

Luego, el corpus de las entrevistas fue sistematizado a través de una matriz, de modo de realizar una lectura horizontal de las 35 entrevistas efectuadas, mediante la técnica propuesta por Spradley(29), basada en el análisis de relaciones semánticas en los dominios culturales (primera y más importante categoría de significados culturales), la construcción de taxonomías (relación entre todos los términos incluidos en los dominios) y análisis de temas (dimensiones de contraste entre los términos).

\section{Resultados}

La interpretación de los distintos enunciados integrados en el CBA permite la identificación de categorías de significación cultural, es decir, "términos cubiertos", los cuales son expresados por los encuestados mediante la mención a "términos incluidos", es decir, categorías menores que se vinculan con aquellas categorías más amplias mediante relaciones semánticas.

El primer dominio descubierto como de particular relevancia es el de "respeto", categoría de significación cultural abordada de forma directa en el instrumento CBA, a través del enunciado (16) "Tratarme con respeto", siendo el único enunciado en obtener el puntaje máximo en la escala de importancia asignada, entre todos los encuestados.

De tal forma, los enunciados (1) "Tratarme como ser individual"; (17) "Escucharme realmente cuando estoy hablando" y (18) "Aceptar mis sentimientos sin juzgarlos" son conectados permanentemente con la categoría de respeto, o con categorías afines, posibles de considerar incluidas en su definición, tales como "comprensión", "reconocimiento de diversidad", "tolerancia" o "empatía".

Dominios culturales:

"Que me llamen por mi nombre", "que me traten como ser humano", "por mi nombre, no por mi número de cama", "que me traten por mi nombre porque nadie es más" (término incluidos), son expresiones de (relación semántica) respeto (término cubierto).

"Respetar para ser respetado", "todos somos personas y merecemos respeto, siempre y con respeto mutuo", "si, asi como yo te respeto como funcionario", "es lo primordial entre seres humanos, independiente de su trabajo", "si uno no es prepotente no debería recibir un maltrato del enfermero", "es fundamental, para que sea recíproco" (término incluidos): son condiciones (relación semántica) para que se dé el respeto (término cubierto).

"Respeto es signo de buena educación", "ser tratada con dignidad y no ser atropellada por personas mal educadas" (términos incluidos) son consecuencia de (relación semántica) la educación (término cubierto).

"El respeto es fundamental y base de una buena comunicación", "saber cómo hablarme" (términos incluidos) son requisito para (relación semántica) la comunicación entre paciente y enfermero (término cubierto).

"No burlarse de mi pensamiento o de no saber algo, sí, porque creo que toda persona se lo merece, independientemente de su edad, sexo o enfermedad" (términos incluidos) son expresión de (relación semántica) tolerancia (término cubierto).

"Que me llamen por mi nombre", "que me traten por mi nombre porque nadie es más o menos que otro, somos todos iguales", "que me traten como ser humano, por mi nombre, no por mi número de cama" (términos incluidos) son expresiones de (relación semántica) reconocimiento de la unicidad (término cubierto).

"Saber diferenciar que somos distintos unos deotros", 
"me gusta que me traten como persona, no que me conozcan por el número de mi cama, "somos todos diferentes y no somos un número dentro de la sociedad, por algo tenemos un nombre y un apellido" (términos incluidos) son expresiones de (relación semántica) respeto (término cubierto).

"Ya que todos pensamos distinto, vemos las cosas diferentes", "que no todos entendemos igual, somos todos distintos", "cada uno tiene distintas falencias $y$ tiene que ser tratado de distinta manera", "saber diferenciar que somos distintos unos de otros" (términos incluidos) son formas de (relación semántica) tolerancia y reconocimiento de la diversidad (término cubierto).

"Se está tratando con personas y no con objetos, pero también es importante no demostrar preferencias o mejores cuidados hacia ciertos pacientes", "me gustaria que siempre me traten bien sin que importe cuánta plata tenga", "es cuando uno es importante para ellos" (términos incluidos) pueden ser formas de (relación semántica) trato equitativo (término cubierto).

"Tratar a un paciente considerándolo con ideas y convicciones (...) terminas siendo uno más del montón" (términos incluidos) son expresiones de (relación semántica) considerar o no la unicidad de las personas (término cubierto).

"Entregar respeto", "escucharme cuando hablo" (términos incluidos) son expresiones de (relación semántica) buena comunicación y trato respetuoso (término cubierto).

"Ser escuchado me hace sentir bien, es lo que todos esperamos", "para mi pasan a ser parte de mi vida", "cuando uno está enfermo lo que más necesita es la escucha", "uno se siente importante cuando alguien me escucha", "uno necesita ser escuchado y comprendido sobre todo en las condiciones en que se encuentra", "es muy importante sentirse apoyada y escuchada" (términos incluidos) son vías para obtener (relación semántica) bienestar (término cubierto).

"Saber cómo me siento y poder ayudar a mi tratamiento", "uno puede prevenir al enfermero de algo que pueda ocurrir conmigo, entonces el enfermero tiene que escuchar", "muchas veces las enfermeras es- tán muy ocupadas haciendo su trabajo y responden a todo que si y luego se les olvida, deben darse el tiempo de atender las inquietudes de los pacientes" (términos incluidos) son vias para (relación semántica) los objetivos terapéuticos (término cubierto).

"Todos necesitamos que se nos respete, aún en condición de enfermos", "aceptar y respetar lo que pienso y siento", "respetar mis ideas" (términos incluidos) son expresiones de (relación semántica) respeto (término cubierto).

"Comprender mis sentimientos y aceptarlos", "se comprende aunque no se acepte, es comprensión y respeto", "deben hacerlo porque uno se pone más sensible estando hospitalizada", "si, porque ellos no están para juzgar, sino para comprender" (términos incluidos) son expresiones de (relación semántica) comprensión (término cubierto).

"No se puede poner en duda el dolor del paciente", "porque nadie sabe por lo que estoy pasando y cuantos problemas tengo además", "tratar de comprender lo que pienso" (términos incluidos) son formas de (relación semántica) lograr empatía (término cubierto).

"Que usen palabras simples para poder entender bien, porque si me hablan como los doctores no entiendo nada", "de esa manera la información obtenida deja tranquilo al paciente", "que no me hablen en forma cientifica, que la respuesta sea clara", "para entender bien las indicaciones en términos que yo conozca", "dar solución, no usar lenguaje técnico, dar información clara de acuerdo al paciente $o$ familiar", "decirte las cosas que uno pueda entender", "es necesario para no perder tiempo, dando dos veces la misma explicación", "sería lo ideal ya que los términos médicos uno no los entiende" (términos incluidos) son requisitos para (relación semántica) aprendizaje y respeto (término cubierto).

"Que me conteste bien, que no me hable golpeado", "responderme bien" (términos incluidos) son parte de (relación semántica) trato respetuoso (término cubierto).

A partir del análisis de estos dominios, se ha llegado a la construcción de tres taxonomías principales: 
a) Signos o expresiones de respeto: las personas entrevistadas enfatizan aspectos como la forma en que se les habla, escucha y observa, el tono de voz, la actitud de interés o preocupación, preguntarles cómo se sienten, esperar su respuesta y responder en concordancia con lo expresado.

b) Condiciones o requisitos para el desarrollo de un trato respetuoso: considerarles como seres individuales que tiene una cosmovisiones diferentes; historias de vida que les hacen tener creencias, mitos y costumbres que valoran, por lo que no se les debe discriminar; un conocimiento que les ha permitido adaptarse a sus condiciones de vida y asumir conductas en salud acordes con estos aspectos.

c) El ejercicio del profesional que respeta: desde la perspectiva del sujeto de cuidado, aparece con mucha intensidad el concepto de ser persona como una unidad indivisible, que responde como un todo a su realidad social y cultural, que el profesional debe respetar. La relevancia de un proceso de enseñanza congruente con sus capacidades, posibilidades y habilidades de un ser en situación e inmerso en su propio mundo, que requiere un lenguaje comprensivo, claro, oportuno, que les muestre opciones para lograr un mejor nivel de salud y bienestar; un cuidado oportuno, accesible y respetuoso, que dé respuesta a sus necesidades, y unas habilidades que les permitan relacionar los distintos elementos involucrados en su situación, para orientarles y guiarles en las mejores prácticas de salud coherentes con su sistema de vida, en lo personal, en lo familiar y en los otros grupos sociales.

\section{Conclusiones}

La información de interés etnográfico analizada permite concluir que el respeto es la primera condición de importancia en el cuidado.

A través del discurso elaborado por los informantes — sujetos de cuidado- se identifican prácticas específicas que, en este contexto sociocultural, el respeto adopta como expresión de facto, la dimensión en que lo moral es llevado al plano de lo concreto en el ámbito profesional de la enfermería. Así también hemos obtenido una aproxima- ción a las condiciones en las cuales se da el trato respetuoso y los elementos constitutivos en que la realización del rol adquiere un significado considerado verdaderamente profesional.

Si consideramos el enfoque etnográfico como una reconstrucción de un escenario o retrato cultural que explora las creencias y costumbres, los resultados nos permiten concluir que existe una grieta entre aquellos aspectos esperados y valorados por los sujetos de cuidado y el cotidiano ejercicio de las(os) enfermeras(os). En el común de las instituciones de salud se presenta una alta demanda de atención, jornadas de trabajo extenuantes, incluso repetitivas en lo que respecta al trabajo en turnos, y que con el paso del tiempo va generando una lista de acciones que conforman una rutina impersonal y escindida de muchas necesidades de las personas, resultando aquello en el desconocimiento del nombre de los sujetos que están siendo cuidados, terminando por reconocerlos por un número de cama o por el mal que les afecta.

Del mismo modo, se genera una réplica en los familiares y amigos de los afectados, quienes son supeditados a un segundo plano, o bien doblegados por las indicaciones expertas. Todos estos hechos se alejan del valor otorgado a los cuidados desde la perspectiva cultural de los propios individuos, para quienes las expectativas están dirigidas precisamente a que sus necesidades y preocupaciones sean escuchadas, ser tratados como personas antes que enfermedades, se respete su privacidad, se les explique su tratamiento(30). Entonces, el cuidado puede verse en el riesgo de centrarse en el hacer, sin observar ni comunicar que se está cuidando, en el solucionar y el administrar, todos aspectos que se encuentran desarticulados del currículo universitario y del objeto de estudio de la enfermería.

Algunos autores $(30,31)$ hacen especial énfasis en potenciar habilidades relacionadas con las dimensiones psicológicas, comunicacionales, relacionales, emocionales, sociales, culturales y familiares. En este sentido, la comprensión y el respeto a la cultura del individuo son ejes tan relevantes del cuidado(13) como el conocimiento fisiológico y psicológico de las personas, por ende la(el) 
enfermera(o) debe comprender que quien se encuentra frente a sí es una persona, un ser humano que merece ser cuidado con respeto y atención. $\mathrm{Si}$ aceptamos esta aseveración como cierta, reconoceremos que el respeto y la dignidad sólo puede ser apreciada comprendiendo qué es lo que estos conceptos significan para el ser humano(32-33).

El valor de cuidar es asignado a aquel brindado con delicadeza, al que optimiza las potencialidades del sujeto, al que asume las debilidades del otro como una experiencia para contribuir al crecimiento y desarrollo personal, independientemente de la condición específica de su salud, al que le prepara para afrontar las consecuencias que la enfermedad tendrá sobre sus capacidades cognitivas, emocionales, sociales y fisiológicas, al que lo apoya durante el proceso para liberarlo gradualmente de éste en la medida en que sus limitaciones van siendo superadas y — desde luego-al cuidado entregado con erudición y movimientos diestros. Aunque este último aspecto, en la perspectiva analizada, no tiene tanta relevancia como los elementos anteriores.

Es interesante observar cómo las personas — con independencia de lo que suele reducirse a la expresión "variables socioculturales" - convergen en reconocer aspectos que les hacen sentirse respetadas y en el valor otorgado a esta dimensión. Si consideramos que el valor de lo justo es porque hemos vivido lo injusto, ¿tendríamos que inferir que la forma en que ellos aprendieron el valor de ser respetados se debe a que durante experiencias anteriores de ser cuidados han sido tratados en forma injusta y desconsiderada? Debemos hacernos cargo de este aspecto en la actualidad y en el futuro, ya que el pasado no lo podemos cambiar. Esta misión debe ser la meta no sólo para el profesional de enfermería sino de todo profesional, en especial quienes participan de las experiencias de salud, pues éstas involucran personas que se encuentran en una etapa de máxima vulnerabilidad, como es el sentir amenazada la existencia en su más amplio sentido.

El trabajo que se ha intentado resumir en este artículo encuentra sus limitaciones en dos aspectos metodológicos. En primer lugar, los informantes fueron agregados a la muestra en una misma institución de salud, por lo cual pudo haber existido cierta homogeneidad en el discurso; es necesario entonces replicar el estudio incorporando una dimensión de diversidad. En segundo, que las entrevistas hayan sido estructuradas a partir de los enunciados de una escala —objeto de validación- puede haber ejercido influencias inconscientes en el pensamiento de los informantes $y$, por ende, en las respuestas; esto debiera ser explorado en el futuro a partir de entrevistas individuales o grupales, semiestructuradas y en profundidad.

De cualquier modo, los resultados abren una discusión sobre las prioridades del trabajo en salud, con énfasis en el foco de atención de enfermería, y plantean una línea para trabajos futuros relacionados con los aspectos intangibles del cuidado, su planificación, documentación y retroalimentación. 


\section{Referencias}

1. Gastmans C. A fundamental ethical approach to nursing: some proposals for ethics education. Nursing Ethics 2002; 9: 494-507.

2. Neville L. Moral difficulties in nursing practice: reflections on the practice of a nurse educator. Nurse Education in Practice 2004; 4(9): 128-134.

3. International Council of Nurses. The ICN Code of Ethics for Nurses. Geneva: Imprimerie Fornara; 2006.

4. Sung K, Kim BJ. Respect for the Elderly: Implications for Human Service Providers. Lanham, MD: University Press of America; 2009.

5. Millares M, Prieto M. Universalidad de la enfermería. Procedente de Primer seminario sobre construcción del conocimiento en enfermería; 2008, septiembre 4-5; Medellín: Universidad de Antioquia, 2008.

6. Rohrbach C. Cuidar es antiguo como el mundo y tan cultural como la diversidad de la humanidad. Cultura de los cuidados 1997; 1(2): 36-39.

7. Siles J. Historia de la enfermería: Una aportación epistemológica desde la perspectiva cultural de los cuidados. Cultura de los cuidados 2008; 12(24): 5-6.

8. Salas M. Una introducción a la naturaleza del cuidado. Cultura de los cuidados 2004; 7(15): 43-48.

9. Watson J. Nursing. Human science and human care: a theory of nursing. Connecticut: AppletonCentury Crofts; 1985.

10. Pfettscher S, Graaf K, Marriner A, Mossman C, Slebodnik M. Florence Nightingale. La enfermería moderna. In: Marriner A, Raile M, (eds.) Modelos y teorías en enfermería. 4a ed. Madrid: Harcout; 2000: 65-83.

11. Zárate R. La gestión del cuidado de enfermería. Index de enfermería 2004; 13(44-45): 21-25.

12. Ramiró A. Valores y actitudes profesionales. Estudio sobre la práctica profesional enfermera en Catalunya (Tesis doctoral) Departamento de Sociología y Análisis de las Organizaciones. Facultad de Ciencias Económicas y Empresariales, Universidad de Barcelona; 2005.

13. Leddy S, Pepper JM. Bases conceptuales de la enfermería profesional. $1^{\text {a }}$ edición. New York: Organización Panamericana de la Salud; 1989.

14. Johansson P, Oléni M, Fridlund B. Patient satisfaction with nursing care in the context of health care: a literature study. Scandinavian Journal of Caring Sciences 2002; 16: 337-334

15. Rehnström L, Christensson L, Leino-Kilpi, Mitra Unosson. Adaptation and psychometric evaluation of the Swedish version of the Good Nursing Care Scale for Patients. Scandinavian Journal of Caring Sciences 2003; 17: 308-314

16. Kvåle K, Bondevik M. What is important for patient centred care? A qualitative study about the perceptions of patients with cancer. Scandinavian Journal of Caring Sciences 2008; 22: 582-589

17. Milton CL. Ethical implications for acting faithfully in the nurse-person relationship. Nursing Science Quarterly 2002; 15: 21-24.

18. Nortvedt P. Needs, closeness and responsibilities. An inquiry into some rival moral considerations in nursing care. Nursing Philosophy 2001; 2:112-121.

19. Brilowski G, Wendler C. An evolutionary concept analysis of caring. Journal of Advanced Nursing 2004; 50(6): 641-650.

20. Swanson KM, Wojnar DM. Optimal healing environment in nursing. Journal of Alternative and Complementary Medicine 2004; 10: 43-48.

21. McCrea MA, Atkinson M, Bloom T, Merkh K, Najera IL, Smith C. The healing energy of relationships. Nursing Administration Quarterly 2003; 27: 240-248.

22. Berg L, Danielson E. Patients' and nurses' experiences of the caring relationship in hospital: an aware striving for trust. Scandinavian Journal of Caring Sciences 2007; 21: 500-506. 
23. Finfgeld-Connett D. Concept synthesis of the art of nursing. Journal of Advanced Nursing 2008; 62(3): 381-388

24. Meiers SJ, Tomlinson PS. Family-nurse co-construction of meaning: a central phenomenon of family caring. Scandinavian Journal of Caring Sciences 2003; 17: 193-201.

25. Cronin SN, Harrison B. Importance of nurse caring behaviors as perceived by patients after myocardial infarction. Heart \& Lung 1988; 17: 374-380.

26. Watson J. Nursing: The Philosophy and Science of Caring. 2a ed. Boulder, Colorado: Associated University Press; 1985.

27. Watson J. Post-hospital nursing: shortage, shifts, and scripts. Nursing Administrative Quarterly 2001; 25(3): 77-82.

28. Watson J, Foster R. The Attending Nurse Caring Model $^{\circledR}$ : integrating theory, evidence and advanced caring-healing therapeutics for transforming professional practice. Journal of Clinical Nursing 2003; 12(3): 360-365.

29. Spradley J. The Ethnographic Interview. New York: Holt, Rinehart and Winston; 1979.

30. Valverde C. Comunicación terapéutica en enfermería. $1^{\text {a }}$ ed. Madrid: DAE; 2007.

31. Ospina B. Técnicas de Comunicación Terapéutica. Medellín: Editorial Universidad Antioquia; 2001.

32. Castledine G. Nursing elderly people with dignity. British Journal of Nursing 1996; 5: 191.

33. Gallagher A. Dignity and respect for Dignity-Two key Health Professional values: Implications for nursing Practice. Nursing Ethics 2004; 11(6): 587-599.

Recibido: 10 de junio de 2010

Aceptado: 15 de octubre de 2010 